\title{
Early rehabilitation after lung transplantation with extracorporeal membrane oxygenation (ECMO) of COVID-19 patient: a case report
}

\author{
Lin Mao", Lunjie Luo", Daming Wang, Ying Yu, Shihao Dong, Pu Zhang, Yun Sun, Zuobing Chen \\ Department of Rehabilitation Medicine, The First Affiliated Hospital of Zhejiang University, Hangzhou, China \\ \#These authors contributed equally to this work. \\ Correspondence to: Zuobing Chen. Department of Rehabilitation Medicine, The First Affiliated Hospital of Zhejiang University, Qingchun Road No. \\ 79, Hangzhou, China. Email: czb1971@zju.edu.cn.
}

\begin{abstract}
Even when severe acute respiratory syndrome coronavirus 2-related coronavirus disease 2019 (COVID-19) is treated with first-line drugs, it progresses and leads to irreversible loss of lung function in some critically ill patients, and lung transplantation is an effective treatment for end-stage chronic pulmonary disease. This case report mainly describes the rehabilitation of a 66-year-old female patient with severe COVID-19 after bilateral lung transplantation. The old patient had a body mass index of $31.2 \mathrm{~kg} / \mathrm{m}^{2}$. She underwent bilateral lung transplantation due to severe and irreversible injury of both lungs. Long-term mechanical ventilation and extracorporeal membrane oxygenation (ECMO) treatment and preoperative and postoperative high-dose corticosteroid therapy and due to the size of the donor lung does not match the size of the recipient's diseased lung, and the right middle lobe of the graft is removed before transplantation. Weaning from the ventilator failed due to weak neuromuscular drive, and muscle strength. A full, personalized pulmonary rehabilitation program was initiated with the help of the physical therapists, the respiratory therapy, the doctors, the nurses and psychotherapist team based on the functional levels. The rehabilitation intervention was conducted on postoperative day 4, This included posture management, airway clearance techniques, respiratory training, muscle strength training, transfer training, daily therapeutic bronchoscopy and psychological support. The ECMO was removed successfully on the fifth day. the patient's physical function, muscle strength and the quality of life has been improved. The good prognosis after rehabilitation indicates that early rehabilitation intervention is effective and feasible and safety for patients after lung transplantation.
\end{abstract}

Keywords: Coronavirus disease 2019 (COVID-19); lung transplantation; early rehabilitation; case

Submitted Nov 25, 2020. Accepted for publication Mar 20, 2021.

doi: 10.21037/atm-21-456

View this article at: http://dx.doi.org/10.21037/atm-21-456

\section{Introduction}

Severe acute respiratory syndrome coronavirus 2 (SARS-CoV-2) is a novel coronavirus, a member of the $\beta$-coronavirus family (1), which can further cause ALI by binding to the angiotensin converting enzyme 2 (ACE2) receptor invading alveolar epithelial cells, small vascular endothelial cells, macrophages, and lymphocytes (2). This process induces the massive release of cytokines and chemokines, which further activates and recruits inflammatory cells such as neutrophils and macrophages to the infected site, releasing the above-mentioned cytokines/chemokines, leading to the infiltration of peripheral lymphocytes into the lungs to phagocyte and kill the virus. The pathological dissection of the lungs of coronavirus disease 2019 (COVID-19) patients showed that the infiltration of lymphocytes in the lungs was mainly scattered with monocytes. The number of lymphocytes was greatly reduced, and the functional state of lymphocytes was overactivated, which resulted in severe damage of the alveolar epithelial cells, causing pathophysiological changes similar to acute respiratory distress syndrome (ARDS) (3).

Patients with COVID-19 usually show respiratory 
symptoms. Mild COVID-19 manifests with symptoms of fever, dry cough, and mild fatigue. Severe COVID-19 is characterized by dyspnea and/or hypoxemia. Critical COVID-19 may rapidly progress to acute respiratory distress syndrome, septic shock, uncorrectable metabolic acidosis, coagulation disorders, and multiple organ failure. The condition of most patients improves after treatment. However, 7-26\% of the patients need to be admitted to intensive care units (ICUs) $(4,5)$. Some of them may develop acute respiratory distress syndrome and require support such as invasive or noninvasive ventilation and extracorporeal membrane oxygenation (ECMO), and some even require lung transplantation. To date, only a few COVID-19 patients in China have received lung transplants.

A patient with pulmonary consolidation secondary to severe COVID-19 received bilateral lung transplants after 15 day of ECMO at the First Affiliated Hospital, School of Medicine, Zhejiang University. In this case, a multidisciplinary team of physiotherapists, respiratory therapists, doctors, nurses and psychotherapists jointly made and implemented a rehabilitation plan for patients after lung transplantation. The program includes exercise training based on the functional grade [including passive range of motion (PROM), active-assistive ROM, active ROM, resistance exercise, sitting exercise leaning against the backrest, and bed cycling, dynamic sitting balance training, endurance training and so on], respiratory training, airway clearance technology, daily therapeutic bronchoscopy, etc. Moreover, physiotherapy interventions in the rehabilitation of chronic critically ill patients with intensive-care-unitacquired muscle weakness may stimulate walking function. It is worth noting that most hospitals in Chinese mainland have no physical therapists involved in the rehabilitation management of ICU after lung transplantation. Therefore, the participation of physiotherapists in our team plays a very important role in the rehabilitation of patients.

The patient had a good prognosis after rehabilitation. The case is presented as follows. We present the following case in accordance with the CARE reporting checklist (available at http://dx.doi.org/10.21037/atm-21-456).

\section{Case presentation}

A 66-year-old female patient with a body mass index (BMI) of $31.2 \mathrm{~kg} / \mathrm{m}^{2}$ tested positive for SARS-CoV-2 nucleic acid on January 31, 2020. A lung computed tomography (CT) scan showed that her lungs were infected with SARSCoV-2. Therefore, a local hospital diagnosed her with
COVID-19. The patient was admitted to our hospital on February 2 due to critical COVID-19. The patient respiratory oxygenation deteriorated progressively and did not improve after treatment with high flow oxygen inhalation and underwent tracheal intubation and mechanical ventilation on February 3 and ECMO on February 16. She tested negative for SARS-CoV-2 nucleic acid in sputum, bronchoalveolar lavage, blood and fecal samples on February 23. Tracheotomy was performed on February 25. After tracheal intubation, tracheotomy, ECMO, and drug therapy, the damage to her lungs was severe and irreversible, and her oxygenation index was still less than $60 \mathrm{mmHg}$, which was life-threatening. Since the patient tested negative for SARS-CoV-2 on 7 consecutive nucleic acid tests and had acceptable vital organ functions and no obvious contraindications for lung transplantation, lung transplantation was considered and approved by the ethics committee. After her family signed informed consent, we registered the patient into the China Organ Transplant Response System (COTRS). Donor lungs were acquired by COTRS protocol. Bilateral allogeneic lung transplantation was performed on March 1 . Since the size of the donor lungs did not match the size of the diseased lungs of the patient, the right middle lobe of the donor lung was resected prior to transplantation. The patient was admitted to an ICU after transplantation.

The patient received postoperative sedatives and analgesics (propofol, butorphanol), immunosuppressants (methylprednisolone, tacrolimus), an antibiotic (voriconazole), and other medications. On postoperative day 3 (March 4, 2020), the oxygenation index significantly decreased, and the chest CT scan showed diffuse consolidation and atelectasis in both lungs and bilateral subcutaneous emphysema in the chest wall (Figure 1A), which might have been caused by an acute rejection reaction. Therefore, the patient was given methylprednisolone pulse therapy for 3 consecutive days. After the therapy, hormone levels gradually reduced. On postoperative day 5, ECMO was removed. A chest CT scan on postoperative day 7 (March 8, 2020) suggested that the patient's condition was better than it had been on postoperative day 3. Mild bilateral pneumothorax and bilateral subcutaneous emphysema in the chest wall were present but were less severe than they had previously been (Figure 1B). A Chest CT on postoperative day 35 (April 5, 2020) showed that the lesions in both lungs were similar and that the bilateral pleural effusions and the collapse of the lower lobes of both lungs were ameliorated (Figure 1C). 

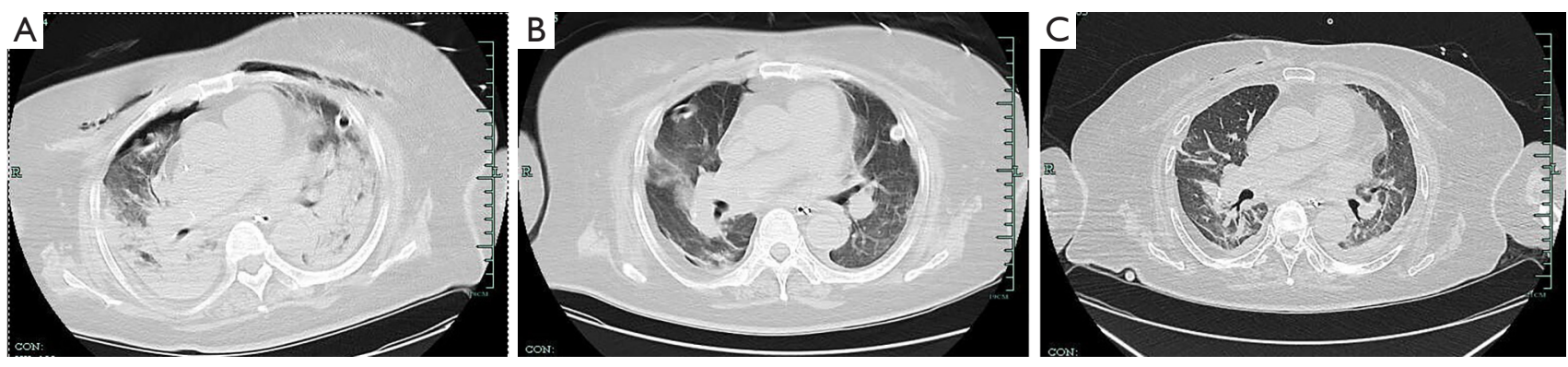

Figure 1 Chest CT images of the patient (from left to right: postoperative days 3, 7, and 35).

Rehabilitation intervention was initiated on postoperative day 4. A comprehensive assessment revealed that the patient's major problems included dyspnea, secretion retention, decreased airway clearance ability, decreased muscle strength, and reduced activities of daily living. The goal of rehabilitation therapy was to relieve dyspnea, improve airway clearance ability, increase muscle strength, and improve activities of daily living. Based on the patient's functional levels (6), a personalized rehabilitation program was developed. The intervention measures included posture management, respiratory training, airway clearance therapy, exercise training, endurance training, strength training, and transfer training. (I) Posture management: ventilation in the prone position was performed in strict accordance with the relevant guidelines. The therapy started on postoperative day 4 and lasted 12 hours each day for 3 consecutive days. Ventilation in the supine and lateral positions were performed intermittently from postoperative day 7. During ventilation in the supine position, the bed was raised by $30^{\circ}-45^{\circ}-60^{\circ}$, and a pillow was placed under the patient's armpit to keep her abdominal muscles fully relaxed. Ventilation therapy in the supine position was performed for 30 minutes each time, 4 times a day. Ventilation therapy in the lateral position was performed once every 2 hours; during the therapy, a wedge pillow was placed under the patient's back, and she alternated between the right lateral position and the left lateral position. (II) Breathing Training: during respirator $y$ training, the patient was in a comfortable position (semirecumbent, at most times), the auxiliary breathing muscles of the neck and shoulders were relaxed, and the patient was instructed to breathe slowly and pay attention to the expansion of the lower thoracic segment. The training was performed for 10 minutes each time, 2 times a day. (III) Airway clearance technique: the patient was taught to use a cycle of active breathing techniques once every hour. In the lateral and seated positions, manual or high-frequency chest wall oscillations were performed by a physiotherapist along the trachea (7) 2 times a day. (IV) Exercise training: during the training, thoracic expansion exercises were integrated with posture management, and the patient was encouraged to take deep breaths and experience bilateral or unilateral proprioceptive stimulation in different parts of the chest (avoiding the incision site and the chest drainage area). (V) Endurance training: endurance training on a bedside cycle ergometer was performed for 20-30 minutes a day. The training intensity was set at 13 to 14 points based on ratings of perceived exertion, i.e., moderate to slightly high intensity. The patient sat with maximum/minimum/moderate assistance or independently on an adjustable bed/chair for 20-30 minutes each time, 2-3 times a day. (VI) Strength training: six to ten repetition maximum (RM) weight training of the upper and lower limbs was performed using the patient's own weight and sandbags. At the end of each set, the interval between 2 sets was determined based on the degree of fatigue and the patient's vital signs. Each training lasted approximately 10-30 minutes, and the training was conducted once or twice a day initially and 3 to 4 times a day during ICU hospitalization. The patient was trained as much as possible as there were no adverse changes in vital signs. (VII) Transfer training: during transfer training, the patient moved from the bed to the bedside, from the bedside to a chair, and from the chair to the bed repeatedly for 30 minutes each time, 2 times a day. And every time the patient was doing exercises, doctors evaluate the safety of patients' rehabilitation. Physiotherapists are responsible for physical rehabilitation and respiratory training. Respiratory therapy is responsible for the adjustment of ventilator parameters during training and daily therapeutic bronchoscopy. Nurses assist physiotherapists in posture management. The psychotherapist is responsible for the patient's psychological condition. Discontinuation of 
Table 1 Physical therapy outcomes before rehabilitation and 16 weeks after rehabilitation

\begin{tabular}{|c|c|c|c|c|c|c|c|}
\hline Time & $\begin{array}{c}\text { S5Q } \\
\text { (points) }\end{array}$ & $\begin{array}{l}\text { MRC-score } \\
\text { (points) }\end{array}$ & $\begin{array}{l}\text { PFIT-s } \\
\text { (points) }\end{array}$ & $\begin{array}{c}\mathrm{MBI} \\
\text { (points) }\end{array}$ & $\begin{array}{l}\text { Cough strength } \\
\text { (level) }\end{array}$ & Respiratory support & $\begin{array}{l}\text { Borg dyspnea } \\
\text { score (points) }\end{array}$ \\
\hline $\begin{array}{l}\text { Before } \\
\text { rehabilitation }\end{array}$ & 4 & 12 & 0 & 20 & 1 & $\begin{array}{c}\text { PC ventilation }\left(\mathrm{FiO}_{2} 25 \% \text {, PEEP } 5 \mathrm{cmH}_{2} \mathrm{O} \text {, }\right. \\
\left.\text { PS } 21 \mathrm{cmH}_{2} \mathrm{O}\right) \mathrm{SPO}_{2} 98 \%\end{array}$ & 5 \\
\hline $\begin{array}{l}16 \text { weeks after } \\
\text { rehabilitation }\end{array}$ & 5 & 42 & 6 & 50 & 3 & $\begin{array}{l}\text { PSV }\left(\mathrm{FiO}_{2} 25 \%, \mathrm{PS} 10 \mathrm{cmH}_{2} \mathrm{O}\right. \\
\left.\text { PEEP } 5 \mathrm{cmH}_{2} \mathrm{O}\right) \mathrm{SPO}_{2} 98 \%\end{array}$ & 2 \\
\hline
\end{tabular}

S5Q, Standardized Five Questions; MRC-s, Medical Research Council score; PFIT-s, Physical Function in ICU Test-Score; MBI, Modified Barthel Index; PC, pressure control; PS, pressure support; PEEP, positive end expiratory pressure; PSV, pressure support ventilation.

rehabilitation were deterioration of subjective symptoms (dyspnea) and significant fluctuations in vital signs, which could further aggravate the patient's symptoms and become life-threatening.

Before rehabilitation and after the completion of rehabilitation, the Standardized Five Questions (S5Q), Borg dyspnea score, coughing ability (8), Modified Barthel Index (MBI), Medical Research Council score (MRC-s) (9), and the Physical Function in ICU Test-Score (PFIT-s) (10) score were used to evaluate physical therapy outcomes. (I) S5Q: the S5Q includes 5 questions. Each question has a maximum score of 1 point, and the maximum total score is 5 points. (II) Borg dyspnea score: 0 points indicate no dyspnea, 1-3 points indicate mild dyspnea, $5-7$ points indicate moderate to severe dyspnea, and $8-10$ points indicate extremely severe dyspnea. (III) Cough strength: The strength of voluntary coughing after gastrectomy was used to evaluate the cough strength, which can be divided into 6 levels: level 0 corresponds to no cough; level 1 corresponds to audible tracheal sound but no cough; level 2 corresponds to audible but weak cough; level 3 corresponds to clearly audible cough; level 4 corresponds to strong cough; and level 5 corresponds multiple sequential strong coughs. (IV) MBI: the MBI assesses the activities of daily living, including eating, bathing, grooming, dressing, bowel control, bladder control, toilet use, chair/bed transfer and mobility, walking, climbing stairs. It comprises 10 items with a possible total score of 100 points: scores $>60$ points indicate mildly dependence and acceptable selfcare ability; 41 to 60 points indicate moderate dependence and limited self-care ability; 0 to 40 points indicate strong dependence and no self-care ability. The MBI has high reliability and validity and is simple to use. (V) The MRC-s is the main method for diagnosing and assessing ICUacquired weakness. It mainly scores the strength of 6 major muscle groups (bilateral wrist extensor muscles, elbow flexor muscles, shoulder abductor muscles, foot dorsiflexor muscles, knee extensor muscles, and hip flexor muscles). Each muscle group is scored according to the level of muscle strength, with a possible total score of 60 points. (VI) The PFIT-s evaluates the physical function, muscle strength, and cardiovascular capacity of patients undergoing long-term tracheal intubation.

Throughout the entire rehabilitation process, the patient's pain score was $2-3$ points. Therefore, pain intensity did not affect the progress of rehabilitation in this situation.

The patient is in tracheostomy state. She can only communicate with us through gestures, facial expressions and body postures, and cannot complete the assessment of psychological scale. During the whole process of rehabilitation, the patient could complete the training activities according to the therapist's instructions, and did not show obvious anxiety or depression. The assessments of physical therapy outcomes before rehabilitation and at 16 weeks after rehabilitation is shown in Table 1 . The patient's MRC-score increased from 12 to 42 points. Her PFIT-s score increased from 0 to 6 points. Her MBI score increased from 20 to 50 points, suggesting that the patient's activities of daily living were improved. Respiratory support was switched from pressure control (PC) ventilation to pressure support ventilation (PSV), with the pressure support (PS) level decreasing from 21 to $10 \mathrm{cmH}_{2} \mathrm{O}$, and the cough strength increased from level 1 to level 3, suggesting that her respiratory function was improved. The timeline for rehabilitation is shown in Figure 2. All procedures performed in studies involving human participants were in accordance with the ethical standards of the First Affiliated Hospital, Zhejiang University School of Medicine and with the Helsinki Declaration (as revised in 2013). Informed consent was obtained from the patient.

\section{Discussion}

Some studies have suggested that lung transplantation 


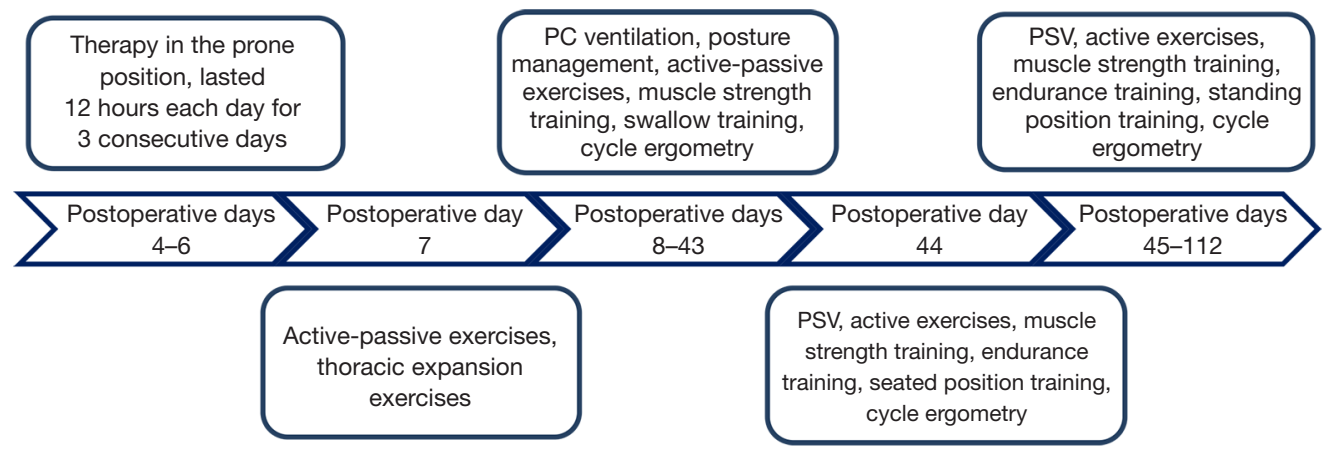

Figure 2 Timeline for rehabilitation. PC, pressure control; PSV, pressure support ventilation.

could be an effective choice for end-stage COVID-19 patients $(11,12)$. Although the respiratory function of lung transplantation survivors is improved, the patient in this article was an elderly obese patient. Long-term mechanical ventilation and ECMO treatment and preoperative and postoperative high-dose corticosteroid therapy and due to the size of the donor lung does not match the size of the recipient's diseased lung, and the right middle lobe of the graft is removed before transplantation all affected her postoperative recovery. Long-term bed rest without early intervention causes muscle atrophy and thus decreased quality of life (13). Survivors of lung transplantation are transferred from the ICU to general wards. Although the effectiveness of rehabilitation in survivors of lung transplantation has been confirmed $(14,15)$, there are few reports regarding early rehabilitation after lung transplantation (6). Therefore, this patient required early rehabilitation to shorten the duration of mechanical ventilation and ICU stay, enhance body function as early as possible, and improve quality of life. After a full evaluation of this case, a multidisciplinary rehabilitation program was created specifically for this difficult patient.

Due to the denervation of transplanted lungs, patients with lung transplantation do not have good airway clearance ability in the early stage, and there are problems with the clearance of airway secretions. Therefore, effective lung management is needed to prevent or reduce infection and improve lung function. It has been reported that in a canine model of single-lung transplantation, ciliary function or mucus characteristics showed significant changes that lasted up to 4 months postoperatively (16). Acute rejection reaction was observed in the patient 3 days after transplantation. A chest CT scan showed diffuse consolidation and atelectasis in both lungs. The patient was then treated with corticosteroid therapy and ventilation in the prone position. Ventilation in the prone position can improve gas exchange and sputum drainage in patients with severe hypoxemia. During this process, patients may experience adverse events such as unplanned catheter removal, wound dehiscence, and wound bleeding, especially in the early stage after cardiothoracic surgery (17), and major thoracic surgery has been considered a contraindication for the prone position. However, a recent single-center nonrandomized trial showed that in the early stage after lung transplantation, the prone position was safe and could significantly improve gas exchange in patients with postoperative refractory hypoxemia (18), and the trial showed no complications by using prone positioning On postoperative day 7 , the patient received airway clearance therapy with a high-frequency oscillator to increase the expiratory flow and improve the clearance rate of airway secretions. Esguerra-Gonzales et al. (19) found that in patients after lung transplantation, airway clearance therapy 4 times a day helps improve peak expiratory flow, the dyspnea score, and oxygen saturation. In patients who underwent lung transplantation, high-frequency chest wall oscillations did not reduce dyspnea scores more significantly than airway clearance therapy did. However, over time, the pulmonary function of patients receiving high-frequency chest wall oscillations generally improved and remained at a level higher than that of patients receiving airway clearance therapy. After rehabilitation, the respiratory support mode was converted from PC ventilation to PSV, and the PS level dropped from 21 to $10 \mathrm{cmH}_{2} \mathrm{O}$, weaning from the ventilator failed. The Borg dyspnea score decreased from 5 to 2 points. Although the cough strength of the patient improved from level 1 to level 3, it did not improve drastically, perhaps because of 2 factors. The first factor 
was the high BMI of this obese patient $\left(31.2 \mathrm{~kg} / \mathrm{m}^{2}\right)$. BMI is an important factor determining respiratory mechanics. Functional residual capacity, lung compliance, and the oxygenation index all decrease with increasing BMI. The second factor is the removal of the right middle lobe of the donor lung due to the size mismatch between the donor lungs and the patient's diseased lungs.

Early infusion tubing, chest drainage tubing, ventilator tubing, ECOM tubing, sedative use, and corticosteroid use restricted the activities of this patient. Therefore, attention should be paid to preventing ICU-acquired muscle weakness. The causes of muscle weakness in patients who have undergone lung transplantation also include the use of calcineurin inhibitors, such as cyclosporin A and tacrolimus. Clinical and animal studies have confirmed the effects of these drugs on the mitochondrial respiratory chain and thus their effects on muscle remodeling (20-22). Therefore, early activity after transplantation is critical to prevent muscle atrophy. Major exercises during the early postoperative period include in-bed passive and active exercises and in-bed cycle ergometry. In-bed, supine cycle ergometry is a treatment strategy for early prevention of deterioration of muscle function after surgery in the ICU and a safe and feasible Even in the case of continuous renal replacement therapy, the rate of adverse events following in-bed cycle ergometry was approximately $0.2 \%$ (23). With the help of doctors, nurses, and physiotherapists, the patient received endurance, strength, and transfer training at appropriate intensities during the later stage. The MRCscore and PFIT-s score showed that after rehabilitation, the patient's motor function improved to some extent. Active lower limb resistance training has been proven to be an effective and feasible treatment method for patients with severe muscle atrophy (24). Because this patient received high doses of corticosteroids and anti-rejection medications before and after transplantation, she was likely to develop osteoporosis and tendon disease. Therefore, excessive flexion and rotation were avoided during resistance training to reduce vertebral compression fractures. At the same time, intermittent exercise was performed to allow the patient to warm up and stretch to avoid tendon injury. Obesity can induce changes in hormones and cytokines and thus leads to increased bone cell apoptosis, decreased osteoblast differentiation, enhanced osteoclast function, and changes in bone marrow microcirculation, resulting in decreased bone mass and bone resorption, which leads to osteoporosis. In the later stage of recovery, an imaging examination of this patient indicated possible compression fractures of the lumbar spine; thus, the exercise intensity was reduced, which is another factor affected her physical recovery.

Long-term tracheotomy can lead to disuse atrophy of the respiratory muscles. Improving the range of motion of the diaphragm muscle, one of the most important respiratory muscles, can improve respiratory efficiency, prevent atelectasis, promote expectoration, and reduce ventilatorassociated pneumonia. During the early postoperative stage, the patient performed thoracic expansion exercises and deep breathing exercises to re-establish lung volume and diaphragm muscle strength (25). Patients with bilateral lung transplantation have a clamshell incision. For these patients, it is recommended that the upper limb flexion should not exceed $90^{\circ}$ during early-stage thoracic expansion exercises.

Patients with COVID-19 experience increased depression and anxiety after isolation treatment (26). The patient is in pneumatectomy state, can only communicate with us through gestures, facial expressions and body postures, and cannot complete the assessment of psychological scale. The patient did not show obviously anxiety or depression. When the patient reported insomnia and nerves, a psychologist was consulted, and sleep medication were prescribed. In addition, we will also play games (such as cards) or family videos with the patient.

Some studies found that patients who recovered from COVID-19 could still test positive for SARS-CoV-2 (27). The patient had an open artificial airway, increasing the risk of infection by health workers at the bedside. In order to ensure that every member of the rehabilitation team is not infected, before working in the isolation ward, in addition to the training of professional knowledge, we also received strict training and practice on the wearing of protective equipment to ensure that every qualified member can work on duty.

In this case, the patient's physical function, respiratory function, and activities of daily living improved after careful evaluation and an individual full rehabilitation program from a multidisciplinary team with early intervention. While ensuring adequate protection, physiotherapists should administer an individualized rehabilitation program based on the evaluation results to reduce the incidence of complications and provide a good foundation for patients to be transferred to general wards or to receive follow-up rehabilitation after hospital discharge.

\section{Acknowledgments}

Funding: This study was supported by the Educational 
Commission of Zhejiang Province, China (No. Y202043413).

\section{Footnote}

Reporting Checklist: The authors have completed the CARE reporting checklist. Available at http://dx.doi.org/10.21037/ atm-21-456

Conflicts of Interest: All authors have completed the ICMJE uniform disclosure form (available at http://dx.doi. org/10.21037/atm-21-456). The authors have no conflicts of interest to declare.

Ethical Statement: The authors are accountable for all aspects of the work in ensuring that questions related to the accuracy or integrity of any part of the work are appropriately investigated and resolved. The study was conducted in accordance with the Declaration of Helsinki (as revised in 2013). The study was approved by Clinical Research Ethics Committee of the First Affiliated Hospital, Zhejiang University School of Medicine (No. IIT20200528A), and informed consent was obtained from the patient.

Open Access Statement: This is an Open Access article distributed in accordance with the Creative Commons Attribution-NonCommercial-NoDerivs 4.0 International License (CC BY-NC-ND 4.0), which permits the noncommercial replication and distribution of the article with the strict proviso that no changes or edits are made and the original work is properly cited (including links to both the formal publication through the relevant DOI and the license). See: https://creativecommons.org/licenses/by-nc-nd/4.0/.

\section{References}

1. Diagnosis and Treatment Protocol for Novel Coronavirus Pneumonia (Trial Version 7). Chin Med J (Engl) 2020;133:1087-95.

2. Zhou P, Yang XL, Wang XG, et al. A pneumonia outbreak associated with a new coronavirus of probable bat origin. Nature 2020;579:270-3.

3. Xu Z, Shi L, Wang Y, Zhang J, Huang L, Zhang C, et al. Pathological findings of COVID-19 associated with acute respiratory distress syndrome. Lancet Respir Med 2020;8:420-2.

4. Wang D, Hu B, Hu C, et al. Clinical Characteristics of
138 Hospitalized Patients With 2019 Novel CoronavirusInfected Pneumonia in Wuhan, China. JAMA 2020;323:1061-9.

5. Guan WJ, Ni ZY, Hu Y, et al. Clinical Characteristics of Coronavirus Disease 2019 in China. N Engl J Med 2020;382:1708-20.

6. Song JH, Park JE, Lee SC, et al. Feasibility of Immediate in-Intensive Care Unit Pulmonary Rehabilitation after Lung Transplantation: A Single Center Experience. Acute Crit Care 2018;33:146-53.

7. Zhang HW, Liu SZ, Zhang ZY, et al. The role of oscillating positive respiratory pressure devices in the treatment of ventilator-associated pneumonia in the intensive care unit. Chinese Journal of Physical Medicine and Rehabilitation 2015;37:45-7.

8. Khamiees M, Raju P, DeGirolamo A, et al. Predictors of extubation outcome in patients who have successfully completed a spontaneous breathing trial. Chest 2001;120:1262-70.

9. Hermans G, Clerckx B, Vanhullebusch T, et al. Interobserver agreement of Medical Research Council sum-score and handgrip strength in the intensive care unit. Muscle Nerve 2012;45:18-25.

10. Denehy L, de Morton NA, Skinner EH, et al. A physical function test for use in the intensive care unit: validity, responsiveness, and predictive utility of the physical function ICU test (scored). Phys Ther 2013;93:1636-45.

11. Han W, Zhu M, Chen J, et al. Lung Transplantation for Elderly Patients With End-Stage COVID-19 Pneumonia. Ann Surg 2020;272:e33-4.

12. Bharat A, Querrey M, Markov NS, et al. Lung transplantation for patients with severe COVID-19. Sci Transl Med 2020;12.

13. Reinsma GD, ten Hacken NH, Grevink RG, et al. Limiting factors of exercise performance 1 year after lung transplantation. J Heart Lung Transplant 2006;25:1310-6.

14. Candemir I, Ergun P, Kaymaz D, et al. The Efficacy of Outpatient Pulmonary Rehabilitation After Bilateral Lung Transplantation. J Cardiopulm Rehabil Prev 2019;39:E7-12.

15. Jastrzebski D, Ochman M, Ziora D, et al. Pulmonary rehabilitation in patients referred for lung transplantation. Adv Exp Med Biol 2013;755:19-25.

16. Tomkiewicz RP, App EM, Shennib H, et al. Airway mucus and epithelial function in a canine model of single lung autotransplantation. Chest 1995;107:261-5.

17. Messerole E, Peine P, Wittkopp S, et al. The pragmatics of prone positioning. Am J Respir Crit Care Med 
2002;165:1359-63.

18. Riera J, Maldonado C, Mazo C, et al. Prone positioning as a bridge to recovery from refractory hypoxaemia following lung transplantation. Interact Cardiovasc Thorac Surg 2017; 25:292-6.

19. Esguerra-Gonzales A, Ilagan-Honorio M, Kehoe P, et al. Effect of high-frequency chest wall oscillation versus chest physiotherapy on lung function after lung transplant. Appl Nurs Res 2014;27:59-66.

20. Sakuma K, Yamaguchi A. The functional role of calcineurin in hypertrophy, regeneration, and disorders of skeletal muscle. J Biomed Biotechnol 2010;2010:721219.

21. Sanchez H, Zoll J, Bigard X, et al. Effect of cyclosporin $\mathrm{A}$ and its vehicle on cardiac and skeletal muscle mitochondria: relationship to efficacy of the respiratory chain. Br J Pharmacol 2001;133:781-8.

22. Zbreski MG, Helwig BG, Mitchell KE, et al. Effects of cyclosporine-A on rat soleus muscle fiber size and phenotype. Med Sci Sports Exerc 2006;38:833-9.

23. Kimawi I, Lamberjack B, Nelliot A, et al. Safety and Feasibility of a Protocolized Approach to In-Bed Cycling

Cite this article as: Mao L, Luo L, Wang D, Yu Y, Dong S, Zhang P, Sun Y, Chen Z. Early rehabilitation after lung transplantation with extracorporeal membrane oxygenation (ECMO) of COVID-19 patient: a case report. Ann Transl Med 2021;9(6):512. doi: 10.21037/atm-21-456
Exercise in the Intensive Care Unit: Quality Improvement Project. Phys Ther 2017;97:593-602.

24. Troosters T, Probst VS, Crul T, et al. Resistance training prevents deterioration in quadriceps muscle function during acute exacerbations of chronic obstructive pulmonary disease. Am J Respir Crit Care Med 2010;181:1072-7.

25. Polastri M, Venturini E, Pastore S, et al. Do Chest Expansion Exercises Aid Re-shaping the Diaphragm Within the First 72 Hours Following Lung Transplantation in a Usual Interstitial Pneumonia Patient? Physiother Res Int 2015;20:191-6.

26. Liu K, Chen Y, Wu D, et al. Effects of progressive muscle relaxation on anxiety and sleep quality in patients with COVID-19. Complement Ther Clin Pract 2020;39:101132.

27. Yuan B, Liu HQ, Yang ZR, et al. Recurrence of positive SARS-CoV-2 viral RNA in recovered COVID-19 patients during medical isolation observation. Sci Rep 2020;10:11887. 